\title{
THE INDEX OF A HOLOMORPHIC MAPPING AND THE INDEX THEOREM
}

\author{
TÔRU ISHIHARA
}

\begin{abstract}
The index theorem for a harmonic mapping of riemannian manifolds is given. Let $f: M \rightarrow N$ be a holomorphic mapping of Kaehler manifolds. Then it is shown that the index of $f$ is zero and that a Jacobi field along $f$ is a holomorphic section of the bundle $f^{*} T(N)$ induced by $f$.
\end{abstract}

1. Introduction. Let $M$ be an $m$-dimensional smooth compact riemannian manifold with or without boundary and $N$ an $n$-dimensional smooth riemannian manifold. We take $M$ to be oriented. Let $f: M \rightarrow N$ be a smooth mapping. In $\$ 2$ we will discuss simply the variational problem of the energy integral $E(f)=\frac{1}{2} \int_{M}\left\|f_{*}\right\|^{2} * 1$. In particular, we describe the second variational formula for a harmonic mapping of $M$ to $N$, which was given by $\mathrm{E}$. Mazet [4] and R. T. Smith [7] when $\partial M=\varnothing$. Applying Smale's index theorem [6], J. Simons got the index theorem for minimal varieties [5]. Similarly, we can get the index theorem for a harmonic mapping.

Let $f: M \rightarrow N$ be a holomorphic mapping of Kaehler manifolds. Then it is a harmonic mapping. In $\$ 3$ we will compute the second variation in the complex case and prove the index of $f$ is zero. Denote by $f^{*} T(N)$ the bundle induced by $f$ from the tangent bundle $T(N)$. A section of $f^{*} T(N)$ is a Jacobi field along $f$ iff it is holomorphic.

2. The index theorem. Let $d s_{M}^{2}=\sum \omega_{i}^{2}$ (resp., $d s_{N}^{2}=\sum \omega_{a}^{* 2}$ ) be a riemannian metric on $M$ (resp., $N$ ), where $\omega_{i}$ (resp., $\omega_{a}^{*}$ ) are local differential forms in $M$ (resp., $N$ ). (Throughout the paper, indices $i, j, k$ (resp., $a, b, c$ ) have the range $1, \ldots, m$ (resp., $1, \ldots, n)$ ) ) We will follow the notation and terminology of [1] in this section. The structure equations of $M$ are given by

$$
d \omega_{i}=\sum \omega_{j} \wedge \omega_{j i}, \quad d \omega_{i j}=\sum \omega_{i k} \wedge \omega_{k j}-\frac{1}{2} \sum R_{j k l}^{i} \omega_{k} \wedge \omega_{l}
$$

Similar equations remain valid in $N$ and we will denote the corresponding quantities by the same notations with asterisks. Let $X$ be a vector field on $M$ with components $X^{i}$. Then $\operatorname{div} X=\sum X_{i}^{i}$, where $X_{j}^{i}$ are the components of the covariant differential of $X$ given by $\sum X_{j}^{i} \omega_{j}=d X^{i}+\sum X^{j} \omega_{j i}$. Let $* 1$ be the canonical riemannian volume form on $M$ and $\omega$ the canonical riemannian

Received by the editors July 5, 1976 and, in revised form, February 18, 1977.

AMS (MOS) subject classifications (1970). Primary 58E15, 53C55.

Key words and phrases. Harmonic mapping, index, holomorphic mapping. 
volume form on the boundary $\partial M$. Now, Green's theorem is described as follows.

$$
\int_{M} \sum X_{i}^{i} * 1=\int_{\partial M} \sum X^{i} \nu^{i} \omega
$$

where $\nu^{i}$ are components of the unit outward normal vector field to the boundary $\partial M$.

Let $f: M \rightarrow N$ be a smooth mapping. Then its differential $f_{*}$ is represented locally as $f^{*}\left(\omega_{a}^{*}\right)=\sum A_{i}^{a} \omega_{i}$. Denote by $A_{i j}^{a}$ the components of the covariant differential of $f_{*}$, that is,

$$
\sum A_{i j}^{a} \omega_{j}=d A_{i}^{a}+\sum A_{j}^{a} \omega_{j i}+\sum A_{a}^{b} \omega_{b a}^{*}
$$

The energy integral of $f$ is given by

$$
E(f)=\frac{1}{2} \int_{M} \sum\left(A_{i}^{a}\right)^{2} * 1 \text {. }
$$

Let $\left\{f_{t}\right\}$ be a variation of $f$, i.e., $f_{t}=f(\cdot, t): M \times I \rightarrow N$ is a smooth mapping with $f_{0}=f$, where $I=[0,1]$. If $f_{t}=f$ on the boundary $\partial M$, it is called a variation fixed on the boundary. In this case the smooth section $U=(\partial f / \partial t)_{0}$ of the induced bundle $f^{*} T(N)$ vanishes on $\partial M$. Let $U^{a}$ be the components of $U$. Using Green's theorem we get

Proposition 1.

$$
\left(\frac{\partial E\left(f_{t}\right)}{\partial t}\right)_{0}=-\int_{M} \sum U^{a} A_{i i}^{a} * 1+\int_{\partial M} \sum U^{a} A_{i}^{a} \nu^{i} \omega .
$$

$f$ is a critical point of $E$ for variations fixed on the boundary if it is harmonic, i.e., $\sum A_{i i}^{a}=0$. Moreover, it is a critical point for all variations if it harmonic with boundary condition $\sum A_{i}^{a} \nu^{i}=0$ on $\partial M$.

Let $f_{t, s}$ be a 2-parameter variation of a harmonic mapping $f: M \rightarrow N$. Put $U=\left(\partial f_{t, s} / \partial t\right)_{0,0}$ and $V=\left(\partial f_{t, s} / \partial s\right)_{0,0} . U_{i}^{a}$ (resp., $\left.U_{i j}^{a}\right)$ are the components of the first (resp., second) covariant differential of $U$. R. T. Smith defined the second variational operator $J_{f}$ by

$$
\left(J_{f} U\right)^{a}=-\left(\sum U_{i i}^{a}+\sum R_{b c d}^{* a} A_{i}^{b} U^{c} A_{i}^{d}\right),
$$

where $R_{b c d}^{* a}$ is the curvature tensor of $N$. Now we have

Proposition 2.

$$
\left(\frac{\partial^{2} E\left(f_{t}, s\right)}{\partial t \partial s}\right)_{0,0}=\int_{M} \sum\left(J_{f} U\right)^{a} V^{a} * 1+\int_{\partial M} \sum V^{a} U_{i}^{a} \nu^{i} \omega
$$

In the case $\partial M=\varnothing$, the above formula was given by E. Mazet [4] and R. T. Smith [7]. Later on in this section we treat only a variation fixed on the boundary. Therefore we assume that the domain of the operator $J_{f}$ is $C_{0}^{\infty}\left(f^{*} T(N)\right)$, the space of sections of $f^{*} T(N)$ vanishing on $\partial M$. An element of $\operatorname{Ker} J_{f}$ is called a Jacobi field along $f$. Let index $(f)$ be the sum of the 
dimensions of the eigenspaces corresponding to negative eigenvalues of $J_{f}$. $\operatorname{Nullity}(f)$ is the dimension of $\operatorname{Ker} J_{f}$.

We will apply Smale's index theorem to our case. We refer to [5] and [6] for the basic facts about the theorem. Let $M$ be a compact manifold with boundary. Let $g_{t}=g(\cdot, t): M \times I \rightarrow M$ be a smooth mapping with $g_{0}=$ identity. $M_{t}$ denotes the images of $M$ under $g_{t} \cdot\left\{g_{t}\right\}$ is called a contraction of $M$ if $M_{t} \subsetneq M_{s}$ whenever $t>s .\left\{g_{t}\right\}$ is said to be of $\varepsilon$-type $(\varepsilon>0)$ if the volume of $M_{t}$ with respect to the induced riemannian metric is less than $\varepsilon$ for every sufficiently large $t$. For every $\varepsilon>0$, there exists a contraction of $\varepsilon$-type. Let $f: M \rightarrow N$ be harmonic. If $\operatorname{Nullity}\left(\left.f\right|_{M_{t}}\right)>0, \partial M_{t}$ is called a conjugate boundary of $f$.

THEOREM 1. Let $M$ be a compact manifold with boundary. Let $f: M \rightarrow N$ be a harmonic mapping. Then there exists $\varepsilon$ such that if $\left\{g_{t}\right\}$ is a contraction of $\varepsilon$-type, the number of conjugate boundaries is finite and

$$
\operatorname{index}(f)=\sum_{0<t<1} \operatorname{Nullity}\left(f_{M_{t}}\right)
$$

Proof. Take normal coordinate systems $\left\{x^{i}\right\}$ and $\left\{y^{a}\right\}$ in neighborhods of an arbitrary point $p$ of $M$ and $f(p)$, respectively. Let $U$ be any section of $f^{*} T(N)$ with components $U^{a}$. Then at $p J_{f}$ has the expression

$$
\left(J_{f} U\right)^{a}=-\sum \frac{\partial^{2} U^{a}}{\partial x_{i}^{2}}-\sum R_{b c d}^{* a} A_{i}^{b} U^{c} A_{i}^{d}
$$

with respect to $\left\{x^{i}\right\}$ and $\left\{y^{a}\right\}$. Using the same argument as in the proof of Proposition 1.2.3 in [5], one can prove that $J_{f}$ is strongly elliptic and has uniqueness in the Cauchy problem. Therefore, the result follows from the main theorem in [6].

3. Jacobi fields along a holomorphic mapping. Let $M$ and $N$ be Kaehler manifolds with dimensions $2 m$ and $2 n$, respectively. The hermitian metric of $M$ (resp., $N$ ) can be written as $d s_{M}^{2}=\sum \omega_{i} \bar{\omega}_{i}$ (resp., $d s_{N}^{2}=\sum \omega_{a}^{*} \bar{\omega}_{a}^{*}$ ) where $\omega_{i}$ (resp., $\omega_{a}^{*}$ ) are local complex-valued linear differential forms of type $(1,0)$ in $M$ (resp., $N$ ). The connection forms $\omega_{i j}$ in $M$ are characterized by the conditions

$$
\begin{aligned}
d \omega_{i} & =\sum \omega_{j} \wedge \omega_{j i}, \quad \omega_{i j}+\bar{\omega}_{j i}=0, \\
d \omega_{i j} & =\sum \omega_{i k} \wedge \omega_{k j}+\sum R_{i k l}^{j} \omega_{k} \wedge \bar{\omega}_{l} .
\end{aligned}
$$

Similar equations are valid in $N$. We will denote the corresponding quantities by the same notations with asterisks.

Assume that $M$ is a compact Kaehler manifold with or without boundary. Let $f: M \rightarrow N$ be a smooth mapping. Then we can put

$$
f^{*}\left(\omega_{a}^{*}\right)=\sum A_{i}^{a} \omega_{i}+\sum A_{i^{*}}^{a} \bar{\omega}_{i}=\sum A_{I}^{a} \omega_{I}
$$

where we use the notations $i^{*}=m+1$ and $\omega_{i *}=\bar{\omega}_{i}$. (In this section, indices 
$I, J, K$ run from 1 to $2 m$.) Set

$$
E^{\prime}(f)=\int_{M} \sum\left(A_{I}^{a}\right)^{2} * 1 \text { and } E^{\prime \prime}(f)=\int_{M} \sum\left(A_{i^{*}}^{a}\right)^{2} * 1 .
$$

Then the energy integral is given by $E(f)=E^{\prime}(f)+E^{\prime \prime}(f)$. Let $A_{I J}^{a}$ be the components of the covariant differential of $f_{*}$. From the definition $f$ is harmonic iff $\sum A_{i i^{*}}^{a}+\sum A_{i^{*} i}^{a}=0$. But since $A_{I J}^{a}$ is symmetric in $I$ and $J$, it is harmonic if $\sum A_{i i^{*}}^{a}=0$ or if $\sum A_{i^{*} i}^{a}=0$. Now $f$ is holomorphic (resp., antiholomorphic) iff $A_{i^{*}}^{a}=0 i$ (resp., $A_{i}^{a}=0$ ). Hence if it is holomorphic or antiholomorphic, it is harmonic.

Let $f_{t}(t \in I)$ be a variation fixed on the boundary. Then we know

Proposition 3 (A. Lichnerowicz). For all $t \in I, d E^{\prime}\left(f_{t}\right) / d t=$ $d E^{\prime \prime}\left(f_{t}\right) / d t$.

Next, we want to study the second variation of $E^{\prime}$. Let $f_{t, s}$ be a 2-parameter variation of a harmonic mapping $f: M \rightarrow N$, i.e., $f_{t, s}=f(\cdot, t, s): M \times I \times I$ $\rightarrow N$ is a smooth mapping with $f_{0,0}=f$ and $f_{t, s}=f$ on $\partial M$. The differential $\left(f_{t, s}\right)_{*}$ is regarded as a $f_{t, s}^{*} T(N)$-valued 1-form on $M \times I \times I$ and it has the local expression

$$
f_{t, s}^{*}\left(\omega_{a}^{*}\right)=\sum B_{I}^{a} \omega_{I}+B_{0}^{a} d t+B_{-1}^{a} d s=\sum B_{\Phi}^{a} \omega_{\Phi}
$$

where $\omega_{0}=d t, \omega_{-1}=d s$. (In this section indices $\Phi, \Psi, \Theta$ have the range $-1,0,1, \ldots, 2 m$.) There is a covariant differential operator naturally induced on the bundle $f_{t, s}^{*} T(N)$. The covariant differential of $\left(f_{t, s}\right)_{*}$ is given by

$$
\sum B_{\Phi \Psi}^{a} \omega_{\Psi}=d B_{\Phi}^{a}-\sum B_{\Psi}^{a} \omega_{\Phi \Psi}+\sum B_{\Phi}^{a} \omega_{b a}
$$

where $\omega_{\Phi 0}=\omega_{0 \Phi}=\omega_{\Phi(-1)}=\omega_{(-1) \Phi}=0$ and $B_{\Phi \Psi}^{a}$ is symmetric in $\Phi, \Psi$. Note that $\left(B_{i}^{a}\right)_{0,0}=A_{i}^{a},\left(B_{i j}^{a}\right)_{0,0}=A_{i j}^{a}$. In addition, $\left(B_{0}^{a}\right)_{0,0},\left(B_{-1}^{a}\right)_{0,0}$ are components of $U=\left(\partial f_{t, s} / \partial t\right)_{0,0}$ and $V=\left(\partial f_{t, s} / \partial s\right)_{0,0}$, respectively.

LEMMA 1. Let $B_{\Phi \Psi \Theta}^{a}$ be the components of the second covariant differential of $\left(f_{t, s}\right)_{*}$. Then we have

$$
\begin{aligned}
B_{\Phi \Psi \Theta}^{a}-B_{\Phi \Theta \Psi}^{a}= & \sum B_{h}^{a} \delta_{\Phi}^{i} R_{i j k}^{h}\left(\delta_{\Psi}^{j} \delta_{\Theta}^{k^{*}}-\delta_{\Theta}^{j} \delta_{\Psi}^{k^{*}}\right) \\
& -\sum B_{\Phi}^{b} R_{b c d}^{* a}\left(B_{\Psi}^{c} \overline{B_{\Theta^{*}}^{d}}-B_{\Theta}^{c} \overline{B_{\Psi^{*}}^{d}}\right),
\end{aligned}
$$

where $0^{*}=0,(-1)^{*}=-1, i^{*}=m+i$ and $(m+i)^{*}=i$.

Proof. Taking the exterior derivative of (2) and using the structure equations in $M$ and $N$, we get the result.

Proposition 4.

$$
\begin{aligned}
\left(\frac{\partial^{2} E^{\prime}}{\partial t \partial s}\right)_{0,0} & =\left(\frac{\partial^{2} E^{\prime \prime}}{\partial t \partial s}\right)_{0,0}=\int_{M}\left(U, J_{f}^{\prime} V\right) * 1 \\
& =\int_{M} \sum\left(U^{a} \overline{\left(J_{f}^{\prime} V\right)^{a}}+\overline{U^{a}}\left(J_{f}^{\prime} V\right)^{a}\right) * 1,
\end{aligned}
$$


where the differential operator $J_{f}^{\prime}: C_{0}^{\infty}\left(f^{*} T(N)\right) \rightarrow C^{\infty}\left(f^{*} T(N)\right)$ is defined to be

$$
\left(J_{f}^{\prime} V\right)^{b}=-\sum V_{i i^{*}}^{b}-\sum A_{i}^{a} R_{a c d}^{* b}\left(\overline{A_{i}^{d}} V^{c}-A_{i^{*}}^{c} \overline{V^{d}}\right) .
$$

Proof. From Proposition 3 it follows that $\partial^{2} E^{\prime} / \partial t \partial s=\partial^{2} E^{\prime \prime} / \partial t \partial s$. Hence we will calculate only $\partial^{2} E^{\prime} / \partial t \partial s$. For any fixed $(u, v) \in I \times I$, let $i_{u v}$ be the injection of $M$ to $M \times I \times I$ such that $i_{u v}(p)=(u, v, p)$ for $p \in M$. Denote by $T_{u v}$ the bundle induced by $f_{t, s} \circ i_{u v}$ from $T(N)$. Then $\left(\partial f_{t, s} / \partial t\right)_{u, v}$ and $\left(\partial f_{t, s} / \partial s\right)_{u, v}$ are smooth sections of $T_{u v}$ with components $\left(B_{0}^{a}\right)_{u, v}$ and $\left(B_{-1}^{a}\right)_{u, v}$, respectively. The differential of $f_{t, s} \circ i_{u v}$ is a $T_{u v}$-valued 1-form with components $B_{I}^{a}$ in the left-hand side of (1). Hence we can put

$$
\begin{aligned}
E^{\prime}\left(f_{t, s}\right) & =E^{\prime}\left(f_{t, s} \circ i_{u v}\right) \\
& =\int_{M} \sum\left|B_{i}^{a}\right|^{2} * 1 .
\end{aligned}
$$

Since

$$
\sum B_{i}^{a} \overline{\partial B_{i}^{a} / \partial t}+\sum \overline{B_{i}^{a}} \partial B_{i}^{a} / \partial t=\sum B_{i}^{a} \overline{B_{i 0}^{a}}+\sum \overline{B_{i}^{a}} B_{i 0}^{a}
$$

we get

$$
\partial E^{\prime} / \partial t=\int_{M} \sum\left(B_{i}^{a} \overline{B_{i 0}^{a}}+\overline{B_{i}^{a}} B_{i 0}^{a}\right) * 1 .
$$

Moreover, we have

$$
\frac{\partial^{2} E^{\prime}}{\partial t \partial s}=\int_{M} \sum\left(B_{i 0}^{a} \overline{B_{i(-1)}^{a}}+\overline{B_{i 0}^{a}} B_{i(-1)}^{a}+B_{i}^{a} \overline{B_{i 0(-1)}^{a}}+\overline{B_{i}^{a}} B_{i 0(-1)}^{a}\right) * 1 .
$$

Using Lemma 1 and Green's theorem, we obtain the desired formula.

LEMMA 2. $J_{f}^{\prime}$ is equal to the operator $J_{f}^{\prime \prime}$ given by

$$
\left(J_{f}^{\prime \prime} V\right)^{b}=-\sum V_{i^{*} i}^{b}-\sum A_{i^{*}}^{a} R_{a c d}^{* b}\left(\overline{A_{i^{*}}^{d}} V^{c}-A_{i}^{c} \overline{V^{d}}\right) .
$$

Proof. If the second variation of $E^{\prime \prime}$ is calculated by the method in the proof of Proposition 4, it follows that $\partial^{2} E^{\prime \prime} / \partial t \partial s=\int_{M}\left(U, J_{f}^{\prime \prime} V\right) * 1$. Hence we get $\int_{M}\left(U, J_{f}^{\prime} V\right) * 1=\int_{M}\left(U, J_{f}^{\prime \prime} V\right) * 1$. This is true for every variation. Thus we obtain $J_{f}^{\prime}=J_{f}^{\prime \prime}$.

From Proposition 4 and Lemma 2 it is evident that a section $U$ of the induced bundle is a Jacobi field iff $J_{f}^{\prime} U=0$ or $J_{f}^{\prime \prime} U=0$.

Let $f: M \rightarrow N$ be a holomorphic mapping. A section $U \in C^{\infty}\left(f^{*} T(N)\right)$ is said to be holomorphic if $U_{i^{*}}^{a}=0$. This condition implies the following: For any point $p \in M$, take local complex coordinate systems $\left\{z_{i}\right\}$ and $\left\{w_{a}\right\}$ in the neighborhoods of $p$ and $f(p)$, respectively. $U$ has the local expression $U=\Sigma U^{a} \partial / \partial w_{a}+\Sigma \overline{U^{a}} \partial / \partial \bar{w}_{a}$. Then it is holomorphic iff $U^{a}$ are always holomorphic functions of the $z_{i}$ 's.

TheOREM 2. Let $f: M \rightarrow N$ be a holomorphic mapping. Then the index of $f$ is zero. A section of $f^{*} T(N)$ vanishing on $\partial M$ is a Jacobi field along $F$ iff it is 
holomorphic. Hence the nullity of $f$ is equal to the dimension of the space of holomorphic sections vanishing on $\partial M$.

Proof. Let

$$
I(U, V)=\int_{M}\left(U, J_{f}^{\prime} V\right) * 1=\int_{M}\left(V, J_{f}^{\prime} U\right) * 1 .
$$

Then it is a symmetric form on $C_{0}^{\infty} f^{*} T(N)$. Since $f$ is holomorphic, $A_{i^{*}}^{a}=0$. Hence $\left(J_{f}^{\prime} U\right)^{b}=\left(J_{f}^{\prime \prime} U\right)^{b}=-\Sigma U_{i^{*} i}^{b}$. Applying the divergence theorem, we get

$$
I(U, U)=-\int_{M} \sum\left(U^{a} \overline{U_{i^{*} i}^{a}}+\overline{U^{a}} U_{i^{*} i}^{a}\right) * 1=2 \int_{M} \sum\left|U_{i^{*}}^{a}\right|^{2} * 1 .
$$

Thus the index of $f$ is zero. $U$ is a Jacobi field iff $U_{i^{*}}^{a}=0$.

For an antiholomorphic mapping, we can obtain a similar result. From the above theorem, it easily follows that a vector field on a Kaehler manifold $M$ is a Jacobi field along the identity mapping of $M$ iff it is holomorphic. For example, let $M$ be a compact Kaehler manifold with negative first Chern class. Then the Nullity of the identity mapping of $M$ is zero.

\section{REFERENCES}

1. S. I. Goldberg and T. Ishihara, Harmonic quasiconformal mappings of riemannian manifolds, Bull. Amer. Math. Soc. 80 (1974), 562-566.

2. S. I. Goldberg, T. Ishihara and N. C. Petridis, Mappings of bounded dilatation of riemannian manifolds, J. Differential Geometry 10 (1975), 619-630.

3. A. Lichnerowicz, Applications harmoniques et variétés kahleriennes, Rend. Sem. Mat. Fis. Milano 39 (1969), 1-12.

4. E. Mazet, La formule de la variation seconde de l'energie au voisinage d'une application harmonique, J. Differential Geometry 8 (1973), 279-296.

5. J. Simons, Minimal varieties in riemannian manifolds, Ann. of Math. (2) 88 (1968), 62-105.

6. S. Smale, On the Morse index theorem, J. Math. Mech. 14 (1965), 1049-1056.

7. R. T. Smith, The second variation formula for harmonic mappings, Proc. Amer. Math. Soc. 47 (1975), 229-236.

Department of Mathematics, Tokushima University, Josanjima, Tokushima 770, Japan 